\title{
Infection deteriorating hepatitis $B$ virus related acute-on-chronic liver failure: a retrospective cohort study
}

Xing-Ran Zhai ${ }^{1}$, Jing-Jing Tong ${ }^{2,3}$, Hong-Min Wang ${ }^{1}$, Xiang $\mathrm{Xu}^{2}$, Xiu-Ying Mu${ }^{1}$, Jing Chen ${ }^{2,3}$, Zi-Feng Liu ${ }^{3}$, Yu Wang ${ }^{3}$, Hai-Bin Su${ }^{2^{*}}$ and Jin-Hua Hu ${ }^{1,2,3^{*}}$ (D)

\begin{abstract}
Background: Infection is common in acute-on-chronic liver failure (ACLF), which may worsen the clinical condition and prognosis. However, the characteristics of infection and its influence on prognosis in hepatitis $B$ virus related ACLF (HBV-ACLF) as defined by the European Association for the Study of the Liver (EASL) have not been clarified. We aimed to investigate the characteristics of infection and its influence on mortality in patients with HBV-ACLF defined by EASL in China.
\end{abstract}

Methods: We performed a retrospective cohort study in patients with HBV-ACLF defined by EASL in a single center from January 2015 to December 2017. These patients were divided into two groups with and without infection.

The incidence, sites of infection, isolated strains, and risk factors associated with mortality were evaluated.

Results: A total of 289 patients were included, among them 185 (64.0\%) were diagnosed with an infection. The most common type of infection was pneumonia (55.7\%), followed by spontaneous bacterial peritonitis (47.6\%) and others. The gram-negative bacteria were the most frequent (58.3\%). Patients with one, two, and three or more infection sites had a gradually increasing incidence of sepsis $(P<0.01)$, septic shock $(P<0.001)$, and ACLF-3 $(P<0.05)$. Also, patients with infection isolated one, two, and three or more strains showed a growing incidence of sepsis $(P<0.01)$ and septic shock $(P<0.001)$. Patients with infection showed a significantly higher 28 -day mortality than those without $(P<0.01)$, especially in patients with ACLF-3. Infection was identified as an independent risk factor for 28-day mortality in all HBV-ACLF patients. Pneumonia and sepsis were identified as independent predictors of 28-day mortality for patients with infection.

Conclusions: Infection is associated with severe clinical course and high mortality in HBV-ACLF defined by EASL. The increased number of infection sites or isolated strains was associated with the occurrence of sepsis and septic shock. Pneumonia and sepsis were independent predictors for mortality in HBV-ACLF patients with infection.

Keywords: Acute-on-chronic liver failure, Hepatitis B virus, Infections, Prognosis

\footnotetext{
*Correspondence: suhaibin302@163.com; 13910020608@163.com

${ }^{2}$ Liver Failure Treatment and Research Center, The Fifth Medical Center of

Chinese PLA General Hospital, Beijing, China

${ }^{1}$ Peking University 302 Clinical Medical School, Beijing, China

Full list of author information is available at the end of the article
} 


\section{Background}

The definition of acute-on-chronic liver failure (ACLF) is different between the eastern and western countries. European Association for the Study of the Liver (EASL) defined ACLF as a severe clinical syndrome characterized by acute insult, organ failures (OFs), and high short-term mortality in patients with liver cirrhosi s[1]. In western countries, alcoholic liver disease is the most common cause of ACLF. However, in the east of Asia, hepatitis B virus (HBV) infection accounts for the overwhelming majority of etiology [2]. Patients with HBVrelated ACLF (HBV-ACLF) are susceptible to infection due to impaired immune function, intestinal barrier dysfunction, and genetic predisposition [3]. Previous studies reported that infection resulted in the progression of ACLF as defined by EASL and even led to higher clinical severity scores and short-term mortality, [4-6] possibly due to the production and release of inflammatory cytokines that led to necrosis and apoptosis of hepatocyte [7]. However, the etiology of these patients is complex and high alcohol consumption accounts for the majority. As demonstrated in a study, [8] the characteristics of HBV-ACLF were markedly distinct from those of alcohol-related ACLF. HBV-ACLF usually manifesting higher clinical severity score, mortality rate, higher incidence of liver failure, lower incidence of kidney failure, and fewer trigger events. Thus, the influence of infection on the prognosis of patients with HBV-ACLF as defined by EASL is still poorly understood.

The objective of this study is to demonstrate the detailed features of infection in patients with HBV-ACLF defined by EASL and explore its influence on the clinical condition and 28-day mortality.

\section{Methods}

\section{Ethical approval}

This study accords with the ethical guidelines of the 1975 Declaration of Helsinki and was approved by the Ethical Committee of the Fifth Medical Center of Chinese People's Liberation Army (PLA) General Hospital (No. 2019016D). Informed consent was waived due to the study was retrospectively designed and the data were anonymous.

\section{Patient selection}

This cohort study consecutively retrospectively included 345 patients with HBV-ACLF hospitalized from January 2015 to December 2017 in the Fifth Medical Center of Chinese PLA General Hospital. Chronic hepatitis B was diagnosed by hepatitis $B$ surface antigen and/or hepatitis $B$ virus deoxyribonucleic acid (HBV-DNA) positivity for $\geq 6$ months [9]. ACLF was diagnosed based on the criteria proposed by the EASL/the American Association for the Study of Liver Diseases [10-12]. The exclusion criteria were as follows: (1) Less than 18 years old; (2) Hepatocellular carcinoma or other malignant tumors; (3) Serious extra-hepatic diseases; (4) Received liver transplantation within 28 days; (5) Lost to follow-up within 28 days; (6) Hospital stay less than 48 h; (7) Pregnancy.

\section{Definitions related to infection}

The diagnostic criteria for bacterial infection were listed as follows. (1) Spontaneous bacterial peritonitis (SBP): neutrophils count $\geq 250 / \mathrm{mm}^{3}$ in ascitic fluid. (2) Pneumonia: clinical manifestations of infection associated with imaging examination showing that new pulmonary infiltration. (3) Urinary tract infection (UTI): high white blood cell (WBC) count (>10/field) was found in urinary sediment accompanied by positive culture results of urine or innumerable WBC per field with negative culture results of urine. (4) Bacteremia: positive blood culture. (5) Skin and soft tissue infection (SSTI): symptoms of infection, such as redness, inflation, high temperature, and pain on the skin. (6) Spontaneous bacterial empyema (SBE): neutrophils number in pleural fluid $\geq 250$ / $\mathrm{mm}^{3}$. (7) Infectious diarrhea: diarrhea with stool microscopic examination showing WBC or routine stool culture showing evidence of pathogenic microorganisms. (8) Cholangitis: right upper abdominal pain, cholestasis, or radiologic evidence of biliary obstruction. (9) Unproven infection: the existence of fever and leukocytosis needs antibiotic treatment with no recognizable sources [13-15].

The criteria used to define fungal infection were as follows. (1) Invasive candidiasis: detection of Candida spp. in the cultures of blood or from other normally aseptic body fluids. (2) Invasive aspergillosis: discovery of Aspergillus by direct laboratory inspection or culture of respiratory specimens when the radiological evidence was consistent with lung infection [16].

Bacteria/fungal infection was grouped as communityacquired (CA), healthcare-associated (HCA), and nosocomial (NS) infections. NS infection referred to an infection that occurred $48 \mathrm{~h}$ after hospitalization. HCA infection was defined as an infection that occurred within $48 \mathrm{~h}$ after hospitalization and met any of the following: (1) Hospitalization or hemodialysis clinic, or intravenous chemotherapy during the past one month; (2) Hospital stay for $\geq 2$ days, or surgery in the past half a year; (3) Residence in a medical center or a long-term care facility. CA infection referred to an infection that occurred within $48 \mathrm{~h}$ after hospitalization and the patients did not satisfy any criteria for HCA infection [17].

Sepsis and septic shock was defined according to Sepsis-3 [18]. Sepsis can be identified by an increase of the sequential organ failure assessment (SOFA) score $\geq 2$ points which is caused by a maladjusted host response to infection. Septic shock, a subset of sepsis in which 
profound circulatory, cellular, and metabolic abnormalities are associated with a higher risk of mortality than with sepsis alone, can be identified by a vasopressor requirement to keep mean arterial pressure (MAP) of 65 $\mathrm{mmHg}$ or higher and serum lactate level higher than 2 $\mathrm{mmol} / \mathrm{L}$ in absent of hypovolemia [18].

\section{Clinical severity scores and HBV-ACLF grade}

The formula for the model for end-stage liver disease (MELD) score was as follows: MELD score $=3.78 \times \ln$ [Total bilirubin $(\mathrm{mg} / \mathrm{dL})]+11.2 \times \ln \quad($ INR $)+9.57 \times \ln$ [Creatinine $(\mathrm{mg} / \mathrm{dL})]+6.43$ [19]. The formula for MELD $\mathrm{Na}$ was as follows: MELDNa $=$ MELD $-\mathrm{Na}-[0.025 \times$ MELD $\times(140-\mathrm{Na})]+140 \quad$ [20]. Child-Turcotte-Pugh (CTP) score was the summation of fractions of the five indexes, including hepatic encephalopathy (HE), ascites, Total bilirubin (TBil), Albumin (Alb), and Prothrombin time prolongation, with 1-3 points for each index, a minimum score of 5 , and a maximum score of 15 [21]. The OFs were evaluated based on the chronic liver failure consortium organ failure score (CLIF-C OFs) system, and chronic liver failure consortium acute-onchronic liver failure score (CLIF-C ACLFs) was computed as follow: CLIF-C ACLFs $=10 \times[0.33 \times$ CLIF-C OFs $+0.04 \times$ Age $+0.63 \times \ln (\mathrm{WBC}$ count $)-2] \quad$ [22]. The SOFA score was assessed as described [23].

ACLF was graded as per the EASL-chronic liver failure consortium acute-on-chronic liver failure in cirrhosis study [10]. ACLF grade-1 (ACLF-1) refers to the existence of single kidney failure, or brain failure accompanied by kidney dysfunction or another single OF accompanied by kidney/brain dysfunction; ACLF grade2 (ACLF-2) refers to the existence of two OFs; ACLF grade-3 (ACLF-3) refers to the existence of three or more OFs.

\section{Statistical analysis}

The data were analyzed using SPSS software (version 24.0, IBM Corporation, Armonk, NY, USA) and plotted using the GraphPad Prism software (version 7.00, GraphPad Software, Inc., La Jolla, CA, USA) and Medcalc software (version 15.2.2, Medcalc Software baba, Ostend, Belgium). Measurement data with normal distribution were expressed as mean \pm standard deviation (SD), and the t-test was used for comparison between two groups or analysis of variance for comparison among three or more groups. Measurement data that did not conform to the normal distribution were represented by the median and interquartile range (IQR), and the Kolmogorov-Smirnov Z rank-sum test was used for comparison between two groups or Kruskal-Wallis Test for comparison among three or more groups. Enumeration data were expressed as number (\%) and intergroup comparison was performed by the chi-square test. Cox proportional hazards regression model was used for univariate and multivariate analysis of 28-day prognostic independent risk factors. Survival curves were estimated by the Kaplan-Meier method and the comparison was performed with the log-rank test. The difference was considered statistically significant if $P<0.05$.

\section{Results}

\section{Baseline characteristics of patients}

A total of 289 patients with HBV-ACLF were included in this study after excluding 56 patients according to the inclusion and exclusion criteria (Fig. 1), among them $236(81.7 \%)$ were males and $53(18.3 \%)$ were females. The mean age was $47.8 \pm 10.9$ years, range from 22 to 76 years old. A total of 185 (64.0\%) patients developed bacterial or fungal infections during the study. Characteristics of HBV-ACLF patients with and without infection are shown in Table 1. Higher WBC count, serum creatinine $(\mathrm{sCr})$, procalcitonin $(\mathrm{PCT})$, lower MAP, $\mathrm{Alb}$, higher incidence of hemorrhage, acute kidney injury (AKI), HE, kidney failure, brain failure, respiratory failure, circulation failure, systemic inflammatory reaction syndrome (SIRS), and higher clinical severity scores including CLIF-C ACLFs, CLIF-C OFs, SOFA scores were presented in patients with infection than non-infection patients.

\section{Characteristics of infection}

The most common type of infection was pneumonia $(55.7 \%, 103 / 185)$, followed by SBP $(47.6 \%, 88 / 185)$, bacteremia $(20.0 \%, 37 / 185)$, UTI $(11.4 \%, 21 / 185)$, SBE $(7.0 \%, 13 / 185)$, and other infections. The most common site of acquisition was NS infection in $94(50.8 \%)$ patients, followed by HCA infection in 76 (41.1\%), and CA infection in 15 (8.1\%).

Positive culture results were detected in 120 among 185 patients with infection. Gram-positive bacteria, gram-negative bacteria, and fungi were identified in 42 (35.0\%), 70 (58.3\%), and 44 (36.7\%) patients, respectively. Patients infected with fungi showed the highest 28-day mortality of $72.7 \%$ compared with 59.5 and $64.3 \%$ in those infected with gram-positive and gram-negative bacteria.

Two hundred and ten isolates were acquired from 120 patients with positive culture results. The most frequent isolated pathogen was gram-negative bacteria in 104 specimens (49.5\%), among which the most common strain was Escherichia coli (18.6\%), the most common source was ascites, followed by Klebsiella pneumoniae (11.4\%), the most common source was ascites, and then Acinetobacter baumannii (5.7\%), the most common source was sputum. Gram-positive bacteria were found in $53(25.2 \%)$ isolates, among which the most frequent strain was Enterococcus faecium (9.0\%), the most 


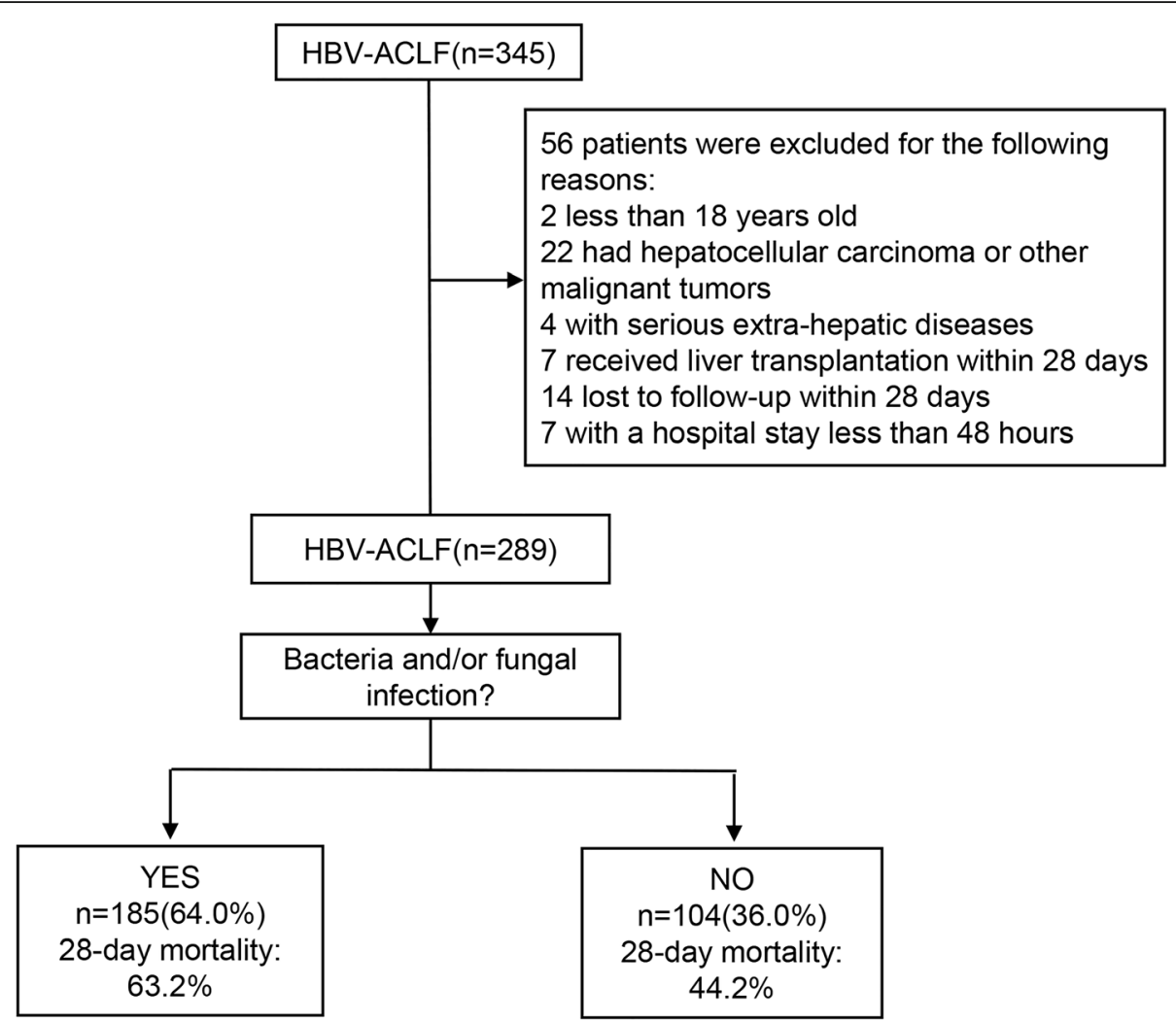

Fig. 1 Flow chart of the patients with HBV-ACLF included and excluded from the study. HBV-ACLF: hepatitis B virus related acute-on-chronic liver failure

frequent source was ascites, followed by Staphylococcus aureus (3.3\%), the most frequent source was sputum, and then Staphylococcus epidermidis (2.9\%), the most frequent source was blood. Fungi were isolated in 53 (25.2\%) specimens, among which Aspergillus fumigatus (11.0\%) was the most prevalent, followed by Candida albicans (8.6\%), the most prevalent source of both was sputum (Table 2).

To study the characteristics of the infection-related index of different type of infection and different pathogens, WBC count, percentage of neutrophils, C-reactive protein (CRP), PCT, the incidence of SIRS, sepsis, septic shock, and ACLF grades were analyzed in patients with single-site infection and single-class pathogen infection. However, there was no statistically significant difference among them (Table 3).

The influence of the increasing number of infection sites and strains on sepsis and septic shock

One hundred and fifteen (62.2\%) presented one infection site, 50 (27.0\%) had two, and 20 (10.8\%) had three or more in $185 \mathrm{HBV}$-ACLF patients with infection. Patients with one, two, three or more infection sites had a gradually increasing incidence of sepsis $(57.4 \%$ vs $78.0 \%$ vs $85.0 \%, P<0.01)$, septic shock (27.0\% vs $48.0 \%$ vs $70.0 \%, P<0.001)$, ACLF-3 $(22.6 \%$ vs $20.0 \%$ vs $35.0 \%, P<0.05)$ and 28 -day mortality $(59.1 \%$ vs $64.0 \%$ vs and $85.0 \%, P>0.05$ )(Table 3 ).

One, two, three or more strains were isolated in 86 (71.7\%), 16 (13.3\%), and 18 (15.0\%) patients, respectively, who showed a gradually increasing incidence of sepsis (57.0\% vs $75.0 \%$ vs $100.0 \%, P<0.01)$, septic shock $(24.4 \%$ vs $56.3 \%$ vs $94.4 \%, P<0.001)$, and 28 -day mortality (60.5\% vs $62.5 \%$ vs $77.8 \%, P>0.05)$ (Table 3$)$.

\section{The influence of infection on 28-day mortality in HBV- ACLF patients}

Patients with infection showed higher 28-day mortality than those without $(63.2 \%$ vs $44.2 \%, P<0.01)$ (Fig. 2 a). On univariate Cox analysis, the presence of infection, AKI, hemorrhage, HE, high WBC count, TBil, international normalized ratio (INR), creatinine, low serum sodium were associated with 28-day mortality. As creatinine and AKI had a strong correlation, creatinine was excluded in the multivariate Cox analysis to avoid collinearity. Multivariate Cox regression analysis showed that the presence of infection ( $\mathrm{HR}=1.515,95 \%$ CI $1.071-$ $2.144, P<0.05)$ was an independent risk factor for 28 day mortality. Other independent risk factors for 28-day 
Table 1 Baseline characteristics of HBV-ACLF patients with and without infection

\begin{tabular}{|c|c|c|c|}
\hline Variables & Infection $(n=185)$ & No infection $(n=104)$ & $P$-value \\
\hline Age (years) & $48.0 \pm 11.0$ & $47.5 \pm 10.6$ & 0.731 \\
\hline Male (\%) & 151 (81.6) & $85(81.7)$ & 0.982 \\
\hline BMI $\left(\mathrm{kg} / \mathrm{m}^{2}\right)$ & $24.9(22.4,27.7)$ & $25.0(22.1,28.1)$ & 0.990 \\
\hline MAP $(\mathrm{mmHg})$ & $85(79,95)$ & $89(81,96)$ & 0.028 \\
\hline HBV-DNA level (IU/ml) & & & 0.427 \\
\hline$\leq 200$ & $41(22.3)$ & $20(19.2)$ & 0.543 \\
\hline $200-2 \times 10^{4}$ & $42(22.8)$ & $33(31.7)$ & 0.098 \\
\hline $2 \times 10^{4}-2 \times 10^{6}$ & $57(31.0)$ & $28(26.9)$ & 0.469 \\
\hline$\geq 2 \times 10^{6}$ & $44(23.9)$ & $23(22.1)$ & 0.729 \\
\hline \multicolumn{4}{|l|}{ Laboratory data } \\
\hline WBC count $\left(10^{9} / L\right)$ & $9.7(6.5,13.1)$ & $7.4(5.5,9.3)$ & $<0.001$ \\
\hline Platelet count $\left(10^{9} / \mathrm{L}\right)$ & $64(40,102)$ & $71(45,108)$ & 0.201 \\
\hline Total bilirubin $(\mu \mathrm{mol} / \mathrm{L})$ & $339 \pm 162$ & $366 \pm 155$ & 0.164 \\
\hline Albumin (g/L) & $28.0(24.0,30.5)$ & $29.0(26.3,32.0)$ & 0.008 \\
\hline $\operatorname{ALT}(U / L)$ & $102(42,243)$ & $96(43,199)$ & 0.881 \\
\hline AST (U/L) & $135(79,278)$ & $128(84,249)$ & 0.855 \\
\hline ALP $(U / L)$ & $124(100,169)$ & $142(110,171)$ & 0.189 \\
\hline GGT (U/L) & $50(33,78)$ & $49(34,74)$ & 0.736 \\
\hline INR & $2.4(1.9,2.8)$ & $2.5(2.0,2.7)$ & 0.214 \\
\hline Creatinine $(\mu \mathrm{mol} / \mathrm{L})$ & $135(90,193)$ & $111(82,147)$ & 0.015 \\
\hline Serum sodium (mmol/L) & $133(129,137)$ & $133(128,137)$ & 0.997 \\
\hline HBeAg (\%) & $97(53.0)$ & $50(48.1)$ & 0.422 \\
\hline CRP (mg/L) & $17.8(9.1,53.9)$ & $12.8(8.4,20.8)$ & 0.050 \\
\hline Procalcitonin (ng/ml) & $1.2(0.5,3.2)$ & $0.6(0.4,1.1)$ & $<0.001$ \\
\hline \multicolumn{4}{|l|}{ Complications (\%) } \\
\hline Ascites & $171(92.4)$ & $94(90.4)$ & 0.545 \\
\hline Hemorrhage & $25(13.5)$ & $6(5.8)$ & 0.041 \\
\hline Acute kidney injury & $107(57.8)$ & $43(41.3)$ & 0.007 \\
\hline $\mathrm{HE}$ & $93(50.3)$ & $65(62.5)$ & 0.036 \\
\hline \multicolumn{4}{|l|}{ Organ failure (\%) } \\
\hline Liver failure & $154(83.2)$ & $93(89.4)$ & 0.153 \\
\hline Coagulation failure & $87(47.0)$ & $60(57.7)$ & 0.090 \\
\hline Kidney failure & $54(29.2)$ & $17(16.3)$ & 0.015 \\
\hline Cerebral failure & $33(17.8)$ & $6(5.8)$ & 0.004 \\
\hline Respiratory failure & $24(13.0)$ & $3(2.9)$ & 0.005 \\
\hline Circulation failure & $22(11.9)$ & $1(1.0)$ & 0.001 \\
\hline \multicolumn{4}{|l|}{ Prognostic score } \\
\hline CLIF-C ACLFs & $48(44,53)$ & $44(40,48)$ & $<0.001$ \\
\hline CLIF-C OFs & $10(10,12)$ & $10(9,11)$ & 0.002 \\
\hline MELD & $30(26,34)$ & $29(25,32)$ & 0.099 \\
\hline MELDNa & $31(28,36)$ & $31(27,34)$ & 0.065 \\
\hline CTP & $13(12,13)$ & $12(11,13)$ & 0.066 \\
\hline SOFA & $9(8,11)$ & $8(6,9)$ & $<0.001$ \\
\hline
\end{tabular}


Table 1 Baseline characteristics of HBV-ACLF patients with and without infection (Continued)

\begin{tabular}{clll}
\hline Variables & Infection $(\boldsymbol{n}=\mathbf{1 8 5})$ & No infection $(\boldsymbol{n}=\mathbf{1 0 4})$ & $\boldsymbol{P}$-value \\
\hline ACLF-1 & $62(33.5)$ & $36(34.6)$ & 0.849 \\
ACLF-2 & $80(43.2)$ & $61(58.7)$ & 0.012 \\
ACLF-3 & $43(23.2)$ & $7(6.7)$ & $<0.001$ \\
SIRS (\%) & $57(30.8)$ & $12(11.5)$ & $<0.001$ \\
\hline
\end{tabular}

Note: All data are expressed as number (\%) or mean \pm SD or median (IQR)

Abbreviations: $H B V-A C L F$ : hepatitis $B$ virus related acute-on-chronic liver failure; $B M I$ : body mass index; $M A P$ : mean arterial pressure; $H B V$-DNA: hepatitis B virus deoxyribonucleic acid; WBC: white blood cells; $A L T$ : alanine aminotransferase; AST: aspartate aminotransferase; $A L P$ : alkaline phosphatase; GGT: gamma-glutamyl transpeptidase; INR: international normalized ratio; HBeAg: hepatitis B virus envelope antigen; CRP: C-reactive protein; HE: hepatic encephalopathy; CLIF-C ACLFs: chronic liver failure consortium acute-on-chronic liver failure score; CLIF-C OFs: chronic liver failure consortium organ failure score; MELD: model for end stage liver disease; CTP: Child-Turcotte-Pugh; SOFA: sequential organ failure assessment; SIRS: system inflammatory reaction syndrome

mortality included the presence of AKI $(\mathrm{HR}=1.875,95 \%$ CI $1.365-2.577, P<0.001), \quad \mathrm{HE} \quad(\mathrm{HR}=1.854,95 \% \quad \mathrm{CI}$ 1.352-2.544, $P<0.001)$, high TBil $(\mathrm{HR}=1.001,95 \% \mathrm{CI}$ $1.000-1.002, P<0.05)$, and INR $(\mathrm{HR}=1.730,95 \% \mathrm{CI}$ $1.472-2.035, P<0.001$ ) (Table 4 ).

Patients with ACLF-1, ACLF-2, and ACLF-3 complicate with infection showed a lower 28-day survival rate than those without infection $(P<0.0001)$ (Fig. 2 b). Among HBV-ACLF grade-3 patients, those with infection showed a significantly higher 28-day mortality than those without $(93.0 \%$ vs $42.9 \%, P<0.01)$. Patients with grade- 1 and -2 showed the same tendency as grade- 3 although there was no significant difference between infection and non-infection group (grade-1: $45.2 \%$ vs $41.7 \%, P=0.737$; grade-2: $61.3 \%$ vs $45.9 \%, P=0.070$ ) (Fig. 2 c).

\section{The influence of pneumonia and sepsis on 28-day mortality in HBV-ACLF patients with infection}

Table 5 shows the predictors associated with 28-day mortality in the Cox regression analysis in $185 \mathrm{HBV}-$ ACLF patients with infection. According to univariate analysis, the presence of pneumonia, SBP, ascites, AKI, HE, SIRS, sepsis, septic shock, elevated WBC, INR were significantly associated with 28-day mortality. As sepsis and septic shock had a strong correlation, septic shock was excluded in the multivariate Cox analysis. In multivariable Cox regression analysis, the presence of pneumonia $(\mathrm{HR}=1.904,95 \% \mathrm{CI} 1.268-2.860, P<0.01)$ and sepsis $(\mathrm{HR}=2.166,95 \% \mathrm{CI} 1.372-3.421, P<0.01)$ were identified as independent predictors for 28-day mortality. Other independent predictors included the presence of AKI $(\mathrm{HR}=1.529,95 \% \mathrm{CI} 1.046-2.235, P<0.05)$, HE $(\mathrm{HR}=1.263,95 \%$ CI 1.087-1.466, $P<0.01)$, and elevated INR $(H R=1.482,95 \%$ CI 1.229-1.788, $P<0.001)$.

\section{Discussion}

ACLF is a clinical syndrome with high short-term mortality. Usually, infection is not only the trigger of ACLF but also a common complication in patients with ACLF. Once an infection occurred, the mortality rate increased significantly $[4,5]$. Excessive inflammation response induced by infection can cause multiorgan failure and death. Compared with the general population, infection is more prone to cause sepsis and septic shock. Therefore, it is vital to identify the characteristics of infection in the management of patients with ACLF. There are many studies about infection in patients with ACLF. But these studies were mainly carried out in western countries. It's well known that the etiology is different between western and eastern countries. In eastern countries, HBV infection is the main cause of ACLF.

Our research is unique because the characteristics and influence of infection on the clinical condition and short-term prognosis were assessed in patients with HBV-ACLF defined by EASL in China. There are four main findings in our research. The first is that pneumonia is the most common form of infection in patients with HBV-ACLF. Gram-negative bacteria were the most frequent cultured microorganisms and Escherichia coli was predominant. Secondly, the infection had a negative influence on the short-term prognosis for HBV-ACLF patients. Thirdly, the increased number of infection sites or isolated strains was associated with an increased risk for sepsis and septic shock. Last but not the least, pneumonia and sepsis can serve as independent predictors for short-term mortality in HBV-ACLF patients with infection.

In our research, the most common type of infection was pneumonia, followed by SBP, bacteremia, UTI, SBE, and others. A previous study [5] in India reported that the most common site of infection was lungs in 172 (45.0\%), followed by SBP in 81 (21.1\%) and UTI in 58 (15.2\%) in patients with ACLF. Another study [15] in China reported that the top three infection sites were the abdominal cavity, respiratory tract, and urinary tract in patients with HBV-ACLF. The results of the above studies were similar to that of our research.

One hundred and twenty patients had positive culture results in 185 patients with infection and 210 strains were isolated. Gram-negative bacteria accounted for the majority of all detected samples and were mostly 
Table 2 Distribution of bacteria and fungi and source of specimens

\begin{tabular}{|c|c|c|c|c|c|c|c|c|}
\hline \multirow[t]{2}{*}{ Microorganisms } & \multirow[t]{2}{*}{ Number } & \multirow{2}{*}{$\begin{array}{l}\text { Constituent } \\
\text { ratio(\%) }\end{array}$} & \multicolumn{6}{|c|}{ Source of specimens $(n=210)$} \\
\hline & & & Ascites & Sputum & Urine & Blood & $\begin{array}{l}\text { pleural } \\
\text { fluid }\end{array}$ & $\begin{array}{l}\text { Other } \\
\text { secretion }\end{array}$ \\
\hline \multicolumn{9}{|l|}{ Gram-positive bacteria $(n=53)$} \\
\hline Enterococcus faecium & 19 & 9.0 & 9 & 2 & 3 & 2 & 1 & 2 \\
\hline Staphylococcus aureus & 7 & 3.3 & 2 & 3 & 1 & 1 & 0 & 0 \\
\hline Staphylococcus epidermidis & 6 & 2.9 & 2 & 0 & 0 & 4 & 0 & 0 \\
\hline Staphylococcus hominis & 5 & 2.4 & 4 & 0 & 0 & 1 & 0 & 0 \\
\hline Enterococcus faecalis & 4 & 1.9 & 2 & 0 & 2 & 0 & 0 & 0 \\
\hline Staphylococcus haemolyticus & 2 & 1.0 & 0 & 1 & 0 & 1 & 0 & 0 \\
\hline Bacillus cereus & 2 & 1.0 & 0 & 0 & 0 & 2 & 0 & 0 \\
\hline Streptococcus pneumoniae & 1 & 0.5 & 0 & 0 & 0 & 1 & 0 & 0 \\
\hline Streptococcus oralis & 1 & 0.5 & 1 & 0 & 0 & 0 & 0 & 0 \\
\hline Bacillus pumilus & 1 & 0.5 & 0 & 0 & 0 & 1 & 0 & 0 \\
\hline Staphylococcus capitis & 1 & 0.5 & 1 & 0 & 0 & 0 & 0 & 0 \\
\hline $\begin{array}{l}\text { Methicillin-resistant coagulase negative } \\
\text { staphylococcus }\end{array}$ & 1 & 0.5 & 1 & 0 & 0 & 0 & 0 & 0 \\
\hline Lactobacillus fermenti & 1 & 0.5 & 1 & 0 & 0 & 0 & 0 & 0 \\
\hline Bacillus vegetabile & 1 & 0.5 & 0 & 0 & 0 & 1 & 0 & 0 \\
\hline Staphylococcus lentus & 1 & 0.5 & 0 & 0 & 0 & 1 & 0 & 0 \\
\hline \multicolumn{9}{|l|}{ Gram-negative bacteria $(n=104)$} \\
\hline Escherichia coli & 39 & 18.6 & 23 & 2 & 1 & 12 & 1 & 0 \\
\hline Klebsiella pneumoniae & 24 & 11.4 & 8 & 6 & 2 & 6 & 1 & 1 \\
\hline Acinetobacter baumannii & 12 & 5.7 & 2 & 7 & 0 & 2 & 1 & 0 \\
\hline Pseudomonas aeruginosa & 8 & 3.8 & 1 & 6 & 0 & 1 & 0 & 0 \\
\hline Stenotrophomonas maltophilia & 8 & 3.8 & 0 & 7 & 0 & 0 & 1 & 0 \\
\hline Klebsiella oxytoca & 3 & 1.4 & 0 & 1 & 1 & 1 & 0 & 0 \\
\hline Chryseobacterium indologenes & 2 & 1.0 & 2 & 0 & 0 & 0 & 0 & 0 \\
\hline Enterobacter aerogenes & 1 & 0.5 & 0 & 1 & 0 & 0 & 0 & 0 \\
\hline Enterobacter asburiae & 1 & 0.5 & 1 & 0 & 0 & 0 & 0 & 0 \\
\hline Raoultella ornithinolytica & 1 & 0.5 & 0 & 0 & 0 & 1 & 0 & 0 \\
\hline Enterobacter cloacae & 1 & 0.5 & 0 & 1 & 0 & 0 & 0 & 0 \\
\hline Raoultella planticola & 1 & 0.5 & 1 & 0 & 0 & 0 & 0 & 0 \\
\hline Burkholderia cepacia & 1 & 0.5 & 0 & 1 & 0 & 0 & 0 & 0 \\
\hline Pseudomonas putida & 1 & 0.5 & 1 & 0 & 0 & 0 & 0 & 0 \\
\hline Flavobacterium meningosepticum & 1 & 0.5 & 0 & 0 & 0 & 1 & 0 & 0 \\
\hline \multicolumn{9}{|l|}{ Fungi $(n=53)$} \\
\hline Aspergillus fumigatus & 23 & 11.0 & 2 & 21 & 0 & 0 & 0 & 0 \\
\hline Candida albicans & 18 & 8.6 & 2 & 12 & 4 & 0 & 0 & 0 \\
\hline Candida tropicalis & 4 & 1.9 & 1 & 3 & 0 & 0 & 0 & 0 \\
\hline Candida parapsilosis & 2 & 1.0 & 0 & 1 & 1 & 0 & 0 & 0 \\
\hline Candida krusei & 2 & 1.0 & 0 & 1 & 1 & 0 & 0 & 0 \\
\hline Candida glabrata & 1 & 0.5 & 0 & 1 & 1 & 0 & 0 & 0 \\
\hline Aspergillus flavus & 1 & 0.5 & 0 & 1 & 0 & 0 & 0 & 0 \\
\hline Mucor & 1 & 0.5 & 0 & 1 & 0 & 0 & 0 & 0 \\
\hline
\end{tabular}


Table 3 Characteristics of the infection-related index of different infection sites and pathogens

\begin{tabular}{|c|c|c|c|c|c|c|c|c|c|c|}
\hline Variables & $\mathbf{N}$ & $\begin{array}{l}\text { WBC } \\
\left(10^{9} / L\right)\end{array}$ & $N(\%)$ & $\begin{array}{l}\text { CRP } \\
(\mathrm{mg} / \mathrm{L})\end{array}$ & $\begin{array}{l}\text { PCT } \\
\text { (ng/ml) }\end{array}$ & SIRS(\%) & Sepsis(\%) & $\begin{array}{l}\text { Septic } \\
\text { shock(\%) }\end{array}$ & $\begin{array}{l}\text { ACLF-1(\%): ACLF-2(\%): } \\
\text { ACLF-3(\%) }\end{array}$ & $\begin{array}{l}\text { 28-day } \\
\text { mortality(\%) }\end{array}$ \\
\hline \multicolumn{11}{|l|}{ Type of infections } \\
\hline $\begin{array}{l}\text { Pneumonia } \\
\text { alone }\end{array}$ & 52 & $\begin{array}{l}10.3(7.3 \\
13.6)\end{array}$ & $\begin{array}{l}82(72, \\
89)\end{array}$ & $\begin{array}{l}16.4(11.0 \\
54.7)\end{array}$ & $\begin{array}{l}1.4(0.8 \\
4.4)\end{array}$ & $\begin{array}{l}16 \\
(30.8)\end{array}$ & $33(63.5)$ & $19(36.5)$ & $\begin{array}{l}10 \text { (19.2): } 25 \text { (48.1): } \\
17(32.7)\end{array}$ & $42(80.8)$ \\
\hline SBP alone & 37 & $\begin{array}{l}9.1(5.8, \\
13.1)\end{array}$ & $\begin{array}{l}79(67, \\
85)\end{array}$ & $\begin{array}{l}17.5(8.8, \\
51.2)\end{array}$ & $\begin{array}{l}1.0(0.5 \\
1.6)\end{array}$ & $\begin{array}{l}14 \\
(37.8)\end{array}$ & $17(45.9)$ & $6(16.2)$ & $\begin{array}{l}12(32.4): 20 \text { (54.1): } \\
5 \text { (13.5) }\end{array}$ & $12(32.4)$ \\
\hline $\begin{array}{l}\text { Bacteraemia } \\
\text { alone }\end{array}$ & 10 & $\begin{array}{l}11.1(6.6, \\
15.5)\end{array}$ & $\begin{array}{l}71(65, \\
82)\end{array}$ & $\begin{array}{l}41.0(24.5 \\
59.2)\end{array}$ & $\begin{array}{l}2.2(1.0, \\
4.6)\end{array}$ & $3(30.0)$ & $6(60.0)$ & $2(20.0)$ & $\begin{array}{l}4(40.0): 4(40.0): \\
2(20.0)\end{array}$ & $6(60.0)$ \\
\hline Others $^{\mathrm{a}}$ & 16 & $\begin{array}{l}8.7(4.8 \\
14.9)\end{array}$ & $\begin{array}{l}73(62 \\
82)\end{array}$ & $\begin{array}{l}26.4(9.4 \\
68.6)\end{array}$ & $\begin{array}{l}1.1(0.5 \\
5.0)\end{array}$ & $5(31.3)$ & $10(62.5)$ & $4(25.0)$ & $\begin{array}{l}5 \text { (31.3): } 9 \text { (56.3): } \\
2 \text { (12.5) }\end{array}$ & $8(50.0)$ \\
\hline \multicolumn{11}{|c|}{ Number of infection sites } \\
\hline One & 115 & $\begin{array}{l}9.9(6.5 \\
13.5)\end{array}$ & $\begin{array}{l}79(68, \\
86)\end{array}$ & $\begin{array}{l}18.9(11.2 \\
53.0)\end{array}$ & $\begin{array}{l}1.4(0.5 \\
2.8)\end{array}$ & $\begin{array}{l}38 \\
(33.0)\end{array}$ & $66(57.4)^{* *}$ & $\begin{array}{l}31 \\
(27.0)^{* * *}\end{array}$ & $\begin{array}{l}31(27.0): 58(50.4): \\
26(22.6)^{*}\end{array}$ & $68(59.1)$ \\
\hline Two & 50 & $\begin{array}{l}9.2(5.1 \\
13.7)\end{array}$ & $\begin{array}{l}81(74, \\
87)\end{array}$ & $\begin{array}{l}19.0(10.9, \\
69.5)\end{array}$ & $\begin{array}{l}1.5(0.6, \\
4.1)\end{array}$ & $\begin{array}{l}17 \\
(34.0)\end{array}$ & $39(78.0)$ & $24(48.0)$ & $\begin{array}{l}22(44.0): 18 \text { (36.0): } \\
10(20.0)\end{array}$ & $32(64.0)$ \\
\hline Three or more & 20 & $\begin{array}{l}12.9(6.9 \\
21.8)\end{array}$ & $\begin{array}{l}81(76, \\
84)\end{array}$ & $\begin{array}{l}55.6(10.1 \\
99.5)\end{array}$ & $\begin{array}{l}0.9(0.7 \\
4.9)\end{array}$ & $8(40.0)$ & $17(85.0)$ & $14(70.0)$ & $\begin{array}{l}9 \text { (45.0): } 4 \text { (20.0): } \\
7 \text { (35.0) }\end{array}$ & $17(85.0)$ \\
\hline \multicolumn{11}{|l|}{ Type of pathogens } \\
\hline G- alone & 42 & $\begin{array}{l}8.9(7.0 \\
12.8)\end{array}$ & $\begin{array}{l}79(70, \\
88)\end{array}$ & $\begin{array}{l}29.3(13.6 \\
73.9)\end{array}$ & $\begin{array}{l}1.6(0.8 \\
4.9)\end{array}$ & $\begin{array}{l}14 \\
(33.3)\end{array}$ & $25(59.5)$ & $13(31.0)$ & $\begin{array}{l}18 \text { (42.9): } 18 \text { (42.9): } \\
6(14.3)\end{array}$ & $25(59.5)$ \\
\hline G+ alone & 26 & $\begin{array}{l}10.7(7.5 \\
15.5)\end{array}$ & $\begin{array}{l}79(74, \\
86)\end{array}$ & $\begin{array}{l}34.0(14.4 \\
87.3)\end{array}$ & $\begin{array}{l}1.5(0.6, \\
2.8)\end{array}$ & $\begin{array}{l}10 \\
(38.5)\end{array}$ & $16(61.5)$ & $5(19.2)$ & $\begin{array}{l}8 \text { (30.8): } 12 \text { (46.2): } \\
6 \text { (23.1) }\end{array}$ & $15(57.7)$ \\
\hline Fungus alone & 23 & $\begin{array}{l}10.3(5.7 \\
15.1)\end{array}$ & $\begin{array}{l}79(69, \\
88)\end{array}$ & $\begin{array}{l}20.4(9.3 \\
55.0)\end{array}$ & $\begin{array}{l}1.5(0.9 \\
4.5)\end{array}$ & $7(30.4)$ & $11(47.8)$ & $4(17.4)$ & $\begin{array}{l}7 \text { (30.4): } 10 \text { (43.5): } \\
6 \text { (26.1) }\end{array}$ & $15(65.2)$ \\
\hline \multicolumn{11}{|l|}{ Number of strains } \\
\hline One & 86 & $\begin{array}{l}9.7(6.6 \\
13.0)\end{array}$ & $\begin{array}{l}79(70, \\
87)\end{array}$ & $\begin{array}{l}27.6(13.5 \\
65.7)\end{array}$ & $\begin{array}{l}1.5(0.9 \\
4.5)\end{array}$ & $\begin{array}{l}29 \\
(33.7)\end{array}$ & $49(57.0)^{* *}$ & $\begin{array}{l}21 \\
(24.4)^{* * *}\end{array}$ & $\begin{array}{l}30 \text { (34.9): } 39 \text { (45.3): } \\
17 \text { (19.8) }\end{array}$ & $52(60.5)$ \\
\hline Two & 16 & $\begin{array}{l}10.0(6.0 \\
14.8)\end{array}$ & $\begin{array}{l}80(72, \\
88)\end{array}$ & $\begin{array}{l}10.9(7.2 \\
71.3)\end{array}$ & $\begin{array}{l}1.1(0.5 \\
2.8)\end{array}$ & $6(37.5)$ & $12(75.0)$ & $9(56.3)$ & $\begin{array}{l}5 \text { (31.3): } 8(50.0): \\
3 \text { (18.8) }\end{array}$ & $10(62.5)$ \\
\hline Three or more & 18 & $\begin{array}{l}12.6(6.5 \\
21.0)\end{array}$ & $\begin{array}{l}82(74, \\
88)\end{array}$ & $\begin{array}{l}55.2(9.6, \\
94.5)\end{array}$ & $\begin{array}{l}1.2(0.5 \\
5.9)\end{array}$ & $6(33.3)$ & $18(100.0)$ & $17(94.4)$ & $\begin{array}{l}5 \text { (27.8): } 5 \text { (27.8): } \\
8 \text { (44.4) }\end{array}$ & $14(77.8)$ \\
\hline
\end{tabular}

Note: All data are expressed as number (\%) or median (IQR)

Abbreviations: $N$ number, WBC white blood cells, N(\%) percentage of neutrophils, CRP C-reactive protein, $P C T$ procalcitonin, SIRS system inflammatory reaction syndrome, $A C L F$ acute-on-chronic liver failure, SBP spontaneous bacterial peritonitis, G- Gram-negative bacteria, G+ Gram-positive bacteria

${ }^{a}$ Other infections included urinary tract infection alone (8): skin and soft tissue infection alone (1): infections diarrhea alone (2): spontaneous bacterial empyema alone (1): the undefined infection alone (4)

${ }^{*} P$-value $<0.05$

${ }^{* *} P$-value $<0.01$

${ }^{* * *} P$-value $<0.001$

Escherichia coli isolated from ascites. There have been studies $[15,24]$ reporting that gram-negative bacteria were the most frequent pathogen in patients with endstage liver disease, which were basically in agreement with the present study, possibly owing to bacterial translocation caused by gut dysbiosis and increased permeability [3, 25]. Thus, early detection and prompt empirical antibiotic treatment are necessary for the management of HBV-ACLF patients with infection.

In our study, HBV-ACLF patients presented overall 28 -day mortality of $56.4 \%$, with a 28 -day mortality of 63.2 and $44.2 \%$ in patients with and without infection, respectively. Infection was identified as an independent risk factor for 28-day survival in patients with HBVACLF. This negative impact was especially evident in patients with HBV-ACLF grade-3, in whom the 28-day mortality of patients with infection was significantly higher than those without. This result was different from that of a study conducted by Fernández et al, [4] which reported that ACLF grade- 1 and grade- 2 patients with infection showed lower 90-day survival than those without, but ACLF grade-3 with and without infections did not show differences in 90-day prognosis. In this study, more than half of the ACLF patients were caused by heavy drinking. The possible explanation for dissimilar results may be the difference in etiologies.

Sepsis refers to the development of organ dysfunction caused by infection. A recent study [26] showed that sepsis was identified as an independent predictor of inhospital mortality and 28-day mortality using Cox 

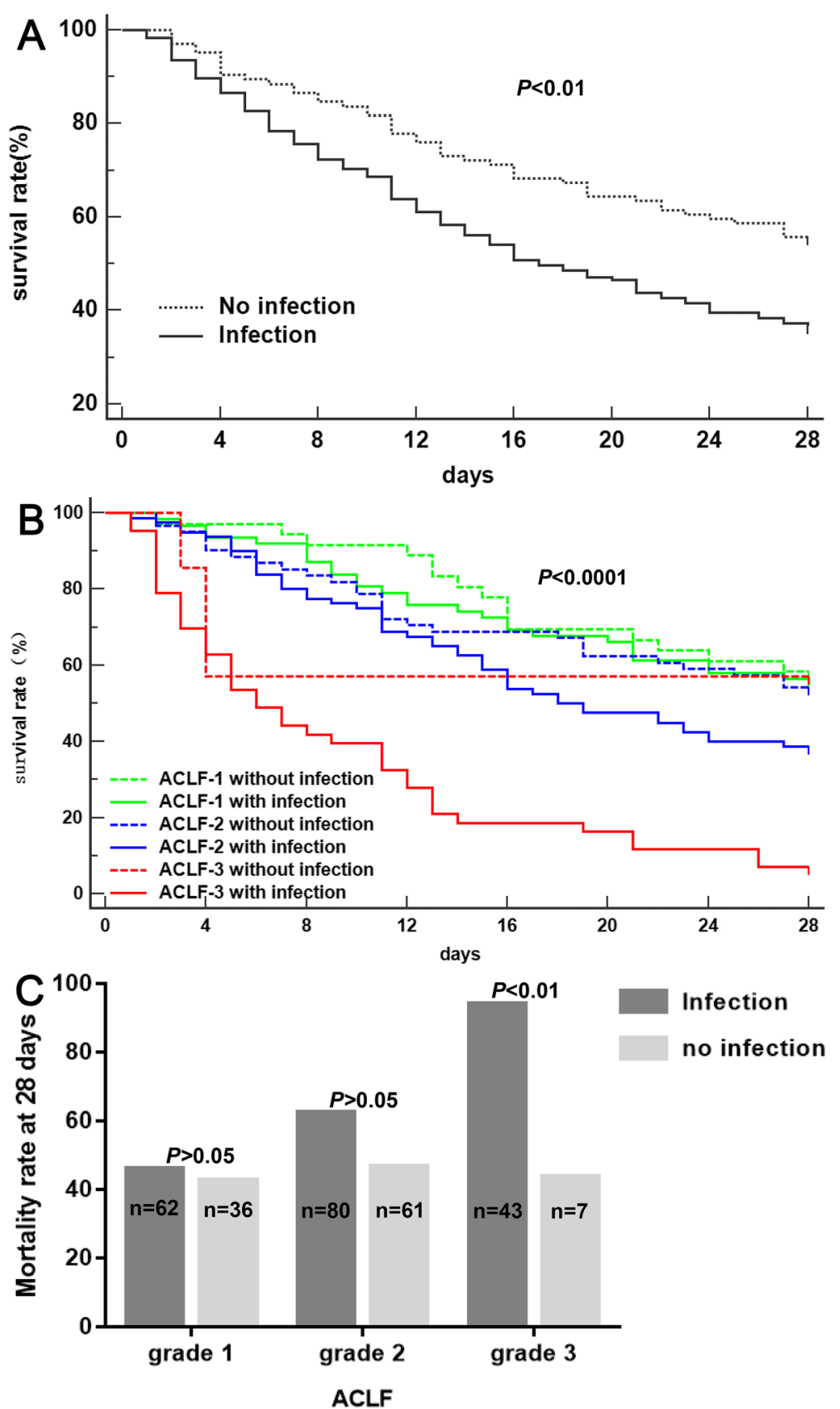

Fig. 2 a Kaplan-Meier survival curves of patients with and without infection. b Kaplan-Meier survival curves of patients with different ACLF grades with and without infection. $\mathbf{c}$ The 28-day mortality rate of patients with different ACLF grades with and without infection

regression analysis in patients with liver cirrhosis and bacterial/fungal infections. Septic shock represents severe circulatory disorders. In our study, increased infection sites and isolated strains were accompanied by an increased incidence of sepsis and septic shock, which undoubtedly complicated the clinical condition and make treatment more challenging. This phenomenon was rarely found in previous studies. Therefore, more attention should be paid to multiple types of infections to avoid deterioration of the clinical course.

Previous research [27] reported that pneumonia and sepsis were independent predictors for 30-day mortality in cirrhosis patients with ascites. Our study found that pneumonia and sepsis were identified as independent predictors for 28-day mortality in HBV-ACLF patients with infection. According to the related literature, 
Table 4 Predictors for 28-day mortality by Cox analysis in patients with HBV-ACLF

\begin{tabular}{|c|c|c|c|c|}
\hline \multirow[t]{2}{*}{ Predictors } & \multicolumn{2}{|l|}{ Univariate analysis } & \multicolumn{2}{|l|}{ Multivariate analysis } \\
\hline & $\mathrm{HR}(95 \% \mathrm{Cl})$ & $P$-value & HR (Cl 95\%) & $P$-value \\
\hline Age (years) & 1.001 (0.986 to 1.015$)$ & 0.934 & & \\
\hline BMI $\left(\mathrm{kg} / \mathrm{m}^{2}\right)$ & 1.022 (0.989 to 1.056$)$ & 0.201 & & \\
\hline MAP (mmHg) & 1.007 (0.992 to 1.022$)$ & 0.376 & & \\
\hline \multicolumn{5}{|l|}{ Laboratory data } \\
\hline WBC count $\left(10^{9} / \mathrm{L}\right)$ & 1.045 (1.020 to 1.071$)$ & $<0.001$ & & \\
\hline Platelet count $\left(10^{9} / \mathrm{L}\right)$ & 0.999 (0.996 to 1.002$)$ & 0.416 & & \\
\hline Total bilirubin $(\mu \mathrm{mol} / \mathrm{L})$ & 1.001 (1.000 to 1.002$)$ & 0.072 & 1.001 (1.000 to 1.002$)$ & 0.019 \\
\hline Albumin(g/L) & 0.992 (0.964 to 1.022) & 0.599 & & \\
\hline INR & 1.694 (1.420 to 2.021$)$ & $<0.001$ & 1.730 (1.472 to 2.035$)$ & $<0.001$ \\
\hline Creatinine $(\mu \mathrm{mol} / \mathrm{L})$ & $1.002(1.000$ to 1.003$)$ & 0.022 & & \\
\hline Serum sodium (mmol/L) & 0.977 (0.953 to 1.001) & 0.062 & & \\
\hline \multicolumn{5}{|l|}{ HBV-DNA level (IU/ml) } \\
\hline$\leq 200$ & 1 & 0.109 & & \\
\hline $200-2 \times 10^{4}$ & 0.779 (0.488 to 1.245$)$ & 0.297 & & \\
\hline $2 \times 10^{4}-2 \times 10^{6}$ & 1.090 (0.705 to 1.685$)$ & 0.699 & & \\
\hline$\geq 2 \times 10^{6}$ & 1.351 (0.867 to 2.107 ) & 0.184 & & \\
\hline \multicolumn{5}{|l|}{ Complications(\%) } \\
\hline Infection & 1.725 (1.225 to 2.427$)$ & 0.002 & 1.515 (1.071 to 2.144$)$ & 0.019 \\
\hline Ascites & 0.868 (0.502 to 1.501$)$ & 0.612 & & \\
\hline Acute kidney injury & 1.549 (1.134 to 2.116$)$ & 0.006 & 1.875 (1.365 to 2.577$)$ & $<0.001$ \\
\hline Haemorrhage & 1.860 (1.205 to 2.871$)$ & 0.005 & & \\
\hline $\mathrm{HE}$ & 1.472 (1.295 to 1.674$)$ & $<0.001$ & 1.854 (1.352 to 2.544$)$ & $<0.001$ \\
\hline
\end{tabular}

Abbreviations: $H B V-A C L F$ hepatitis $B$ virus related acute-on-chronic liver failure, $H R$ hazard ratio, $B M I$ body mass index, MAP mean arterial pressure, WBC white blood cells, INR international normalized ratio, HBV-DNA hepatitis B virus deoxyribonucleic acid, $H E$ hepatic encephalopathy

tracheal intubations [28] and blood products transfusions [29] are considered to be risk factors for pneumonia. Thus, it is imperative to carefully monitor the signs of pneumonia and avoid the related risk factors for early prevention and timely treatment.

Additionally, it has been reported that ACLF patients with fungal infection had a high risk of death [30, 31]. A study performed by Verma et al [30] showed that ACLF patients with invasive fungal infections (IFI) had higher in-hospital mortality than those without IFI $(76.9 \%$ vs $20 \%, P<0.001)$. Lin et al [31] reported that the 12-week mortality was higher in HBV-ACLF patients with IFI as compared to those without $(40.0 \%$ vs $12.1 \%, P<0.001)$. Our study also found that patients with fungal infections had a higher mortality rate than those without $(72.7 \%$ vs $53.5 \%, P<0.05$, see supplementary table). Besides pneumonia and sepsis, we also found that AKI, HE, and INR were independent predictors for 28-day mortality in HBV-ACLF patients with infection. A study performed by Zang et al [32] showed that the development of AKI was a predictor of 180-day survival in ACLF patients using the proportional sub-distribution hazards model.
Besides, a recent study [33] showed that ACLF patients with AKI had a significantly higher 30-day and 90 -day mortality than those without $(79.6 \%$ vs 41.1 , $82.7 \%$ vs $56.7 \%, P<0.05)$. The results of the above researches are consistent with the results of our study. $\mathrm{HE}$, one of the most common severe complications of ACLF, may cause brain edema and intracranial hypertension, which contribute to the progression of ACLF. INR reflects liver coagulation function and severity of liver necrosis.

Our study indeed had three main limitations. Firstly, the history of invasive manipulation and the use of glucocorticoids before infection were not clear in our database, which may be associated with the occurrence of infection. Secondly, this study was retrospectively designed, and it only involved a single center with relatively limited sample numbers, thus a prospective, multi-center study with a larger sample size on HBV-ACLF patients is necessary to further confirm the results of our study. Thirdly, the relation of HBV treatment status and HBV load to infection was not assessed. 
Table 5 Predictors for 28-day mortality by Cox analysis in HBV-ACLF patients with infection

\begin{tabular}{|c|c|c|c|c|}
\hline \multirow[t]{2}{*}{ Predictors } & \multicolumn{2}{|l|}{ Univariate analysis } & \multicolumn{2}{|l|}{ Multivariate analysis } \\
\hline & $\mathrm{HR}(95 \% \mathrm{Cl})$ & $P$-value & $\mathrm{HR}(95 \% \mathrm{Cl})$ & $P$-value \\
\hline Age (years) & 0.996 (0.980 to 1.013$)$ & 0.676 & & \\
\hline BMI $\left(\mathrm{kg} / \mathrm{m}^{2}\right)$ & 1.028 (0.979 to 1.078$)$ & 0.268 & & \\
\hline MAP (mmHg) & 1.001 (0.985 to 1.018$)$ & 0.901 & & \\
\hline \multicolumn{5}{|l|}{ Laboratory data } \\
\hline WBC count $\left(10^{9} / \mathrm{L}\right)$ & 1.038 (1.010 to 1.066$)$ & 0.007 & & \\
\hline Platelet count $\left(10^{9} / \mathrm{L}\right)$ & 0.998 (0.995 to 1.002 ) & 0.306 & & \\
\hline Total bilirubin $(\mu \mathrm{mol} / \mathrm{L})$ & 1.000 (0.999 to 1.001$)$ & 0.498 & & \\
\hline Albumin(g/L) & 0.998 (0.967 to 1.030$)$ & 0.913 & & \\
\hline INR & 1.755 (1.455 to 2.118 ) & $<0.001$ & $1.482(1.229$ to 1.788$)$ & $<0.001$ \\
\hline Creatinine $(\mu \mathrm{mol} / \mathrm{L})$ & 1.001 (1.000 to 1.003$)$ & 0.105 & & \\
\hline Serum sodium (mmol/L) & 0.983 (0.955 to 1.011$)$ & 0.233 & & \\
\hline \multicolumn{5}{|l|}{ HBV-DNA level (IU/ml) } \\
\hline$\leq 200$ & 1 & 0.126 & & \\
\hline $200-2 \times 10^{4}$ & 0.599 (0.341 to 1.051$)$ & 0.074 & & \\
\hline $2 \times 10^{4}-2 \times 10^{6}$ & 0.864 (0.528 to 1.415$)$ & 0.562 & & \\
\hline$\geq 2 \times 10^{6}$ & 1.151 (0.694 to 1.911$)$ & 0.586 & & \\
\hline SIRS & 1.658 (1.136 to 2.421$)$ & 0.009 & & \\
\hline \multicolumn{5}{|l|}{ Type of infection(\%) } \\
\hline Pneumonia & 2.315 (1.563 to 3.429$)$ & $<0.001$ & 1.904 (1.268 to 2.860$)$ & 0.002 \\
\hline SBP & 0.623 (0.429 to 0.904$)$ & 0.013 & & \\
\hline Bacteremia & 1.260 (0.815 to 1.949$)$ & 0.299 & & \\
\hline \multicolumn{5}{|l|}{ Number of infection sites } \\
\hline One & 1 & 0.132 & & \\
\hline Two & 1.184 (0.778 to 1.803$)$ & 0.430 & & \\
\hline Three or more & 1.718 (1.008 to 2.926$)$ & 0.046 & & \\
\hline \multicolumn{5}{|c|}{ Number of isolated microorganisms } \\
\hline Zero & 1 & 0.638 & & \\
\hline One & 0.941 (0.625 to 1.418$)$ & 0.773 & & \\
\hline Two & 0.911 (0.456 to 1.819$)$ & 0.791 & & \\
\hline Three or more & 1.375 (0.749 to 2.524$)$ & 0.304 & & \\
\hline \multicolumn{5}{|l|}{ Complications(\%) } \\
\hline Ascites & 0.777 (0.585 to 1.031$)$ & 0.081 & & \\
\hline Hemorrhage & 1.447 (0.885 to 2.367$)$ & 0.141 & & \\
\hline Acute kidney injury & 1.456 (1.001 to 2.117$)$ & 0.049 & 1.529 (1.046 to 2.235$)$ & 0.028 \\
\hline HE & 1.409 (1.214 to 1.636$)$ & $<0.001$ & 1.263 (1.087 to 1.466$)$ & 0.002 \\
\hline Sepsis(\%) & 2.740 (1.756 to 4.273$)$ & $<0.001$ & $2.166(1.372$ to 3.421$)$ & 0.001 \\
\hline Septic shock(\%) & $2.322(1.612$ to 3.345$)$ & $<0.001$ & & \\
\hline
\end{tabular}

Abbreviations: $H B V-A C L F$ hepatitis B virus related acute-on-chronic liver failure, $H R$ hazard ratio, $B M I$ body mass index, MAP mean arterial pressure, WBC white blood cells, INR international normalized ratio, HBV-DNA hepatitis B virus deoxyribonucleic acid, SIRS system inflammatory reaction syndrome, SBP spontaneous bacterial peritonitis, $H E$ hepatic encephalopathy

\section{Conclusions}

Pneumonia is the most common type of infection in HBV-ACLF patients. Gram-negative bacteria accounted for the most majority of cultured microorganisms, especially Escherichia coli. Infection was associated with increased mortality in HBV-ACLF patients. The increased incidence of sepsis and septic shock was significantly associated with an increased number of infection 
sites and cultured strains. Pneumonia and sepsis were independent predictors for short-term mortality in HBV-ACLF patients with infection. Clinical physicians should pay more attention to infections, especially pneumonia, sepsis, and multiple infections to avoid the progression of HBV-ACLF.

\section{Supplementary information}

Supplementary information accompanies this paper at https://doi.org/10. 1186/s12876-020-01473-y.

Additional file 1 Supplementary table Mortality at 28 days of HBVACLF patients with and without fungal infections.

\section{Abbreviations}

ACLF: Acute-on-chronic Liver failure; HBV: Hepatitis B virus; HBV-ACLF: HBVrelated ACLF; EASL: European Association for the Study of the Liver; PLA: People's Liberation Army; HBV-DNA: Hepatitis B virus deoxyribonucleic acid; SBP: Spontaneous bacterial peritonitis; UTI: Urinary tract infection; WBC: White blood cell; SSTI: Skin and soft tissue infection; SBE: Spontaneous bacterial empyema; CA: Community-acquired; HCA: Healthcare-associated; NS: Nosocomial; SOFA: Sequential organ failure assessment; MAP: Mean arterial pressure; MELD: Model for end-stage liver disease; CTP: ChildTurcotte-Pugh; HE: Hepatic encephalopathy; TBil: Total bilirubin; Alb: Albumin; OFs: Organ failures; CLIF-C OFs: Chronic liver failure consortium organ failure score; CLIF-C ACLFs: Chronic liver failure consortium acute-onchronic liver failure score; SD: Standard deviation; IQR: Interquartile range; sCr: Serum creatinine; PCT: Procalcitonin; AKI: Acute kidney injury; SIRS: Systemic inflammatory reaction syndrome; CRP: C-reactive protein: INR: International normalized ratio; IFI: Invasive fungal infections

\section{Acknowledgments}

Not applicable.

\section{Authors' contributions}

$Z X R, H J H$, and SHB designed this study. ZXR, TJJ, WHM, XX, MXY, CJ, LZF, and WY participated in data collection and analysis. ZXR wrote the first draft with assistance from TJJ, SHB, and $\mathrm{HJH}$. SHB and $\mathrm{HJH}$ edited the final draft. All authors read and approved the final manuscript.

\section{Funding}

No funding.

\section{Availability of data and materials}

The datasets used and/or analyzed during the current study are available from the corresponding author on reasonable request.

\section{Ethics approval and consent to participate}

This study accords with the ethical guidelines of the 1975 Declaration of Helsinki and was approved by the Ethical Committee of the Fifth Medical Center of Chinese PLA General Hospital (No. 2019016D). Informed consent was waived due to the study was retrospectively designed and the data were anonymous.

\section{Consent for publication}

Not applicable.

\section{Competing interests}

The authors declare that they have no competing interests.

\section{Author details}

${ }^{1}$ Peking University 302 Clinical Medical School, Beijing, China. ${ }^{2}$ Liver Failure Treatment and Research Center, The Fifth Medical Center of Chinese PLA General Hospital, Beijing, China. ${ }^{3}$ Medical School of Chinese PLA, Beijing, China.
Received: 28 June 2020 Accepted: 25 September 2020

Published online: 29 September 2020

\section{References}

1. Hernaez R, Sola E, Moreau R, Gines P. Acute-on-chronic liver failure: an update[J]. Gut. 2017;66(3):541-53.

2. Gu WY, Xu BY, Zheng X, Chen J, Wang XB, Huang Y, et al. Acute-on-chronic liver failure in China: rationale for developing a patient registry and baseline characteristics. Am J Epidemiol. 2018;187(9):1829-39.

3. Ekpanyapong S, Reddy KR. Infections in cirrhosis [J]. Curr Treat Options Gastroenterol. 2019:17(2):254-70.

4. Fernandez J, Acevedo J, Wiest R, Gustot T, Amoros A, Deulofeu C, et al. Bacterial and fungal infections in acute-on-chronic liver failure: prevalence, characteristics and impact on prognosis [J]. Gut. 2018;67(10):1870-80.

5. Shalimar R. G, Jadaun SS, Ranjan G, Kedia S, Gunjan D, et al. prevalence, predictors and impact of bacterial infection in acute on chronic liver failure patients [J]. Dig Liver Dis. 2018;50(11):1225-31.

6. Karvellas CJ, Pink F, McPhail M, Austin M, Auzinger G, Bernal W, et al. Bacteremia, acute physiology and chronic health evaluation II and modified end stage liver disease are independent predictors of mortality in critically ill nontransplanted patients with acute on chronic liver failure. Crit Care Med. 2010:38(1):121-6.

7. Bernal W, Auzinger G, Dhawan A, Wendon J. Acute liver failure [J]. Lancet. 2010;376(9736):190-201.

8. Wu T, Li J, Shao L, Xin J, Jiang L, Zhou Q, et al. Development of diagnostic criteria and a prognostic score for hepatitis B virus-related acute-on-chronic liver failure. Gut. 2018;67(12):2181-91.

9. Hou J, Wang G, Wang F, Cheng J, Ren H, Zhuang H, et al. Guideline of prevention and treatment for chronic hepatitis B (2015 update). J Clin Trans Hepatol. 2017;5(4):297-318.

10. Moreau R, Jalan R, Gines P, Pavesi M, Angeli P, Cordoba J, et al. Acute-onchronic liver failure is a distinct syndrome that develops in patients with acute decompensation of cirrhosis. Gastroenterology. 2013;144(7):1426-37.

11. EASL. Clinical practice guidelines for the management of patients with decompensated cirrhosis. J Hepatol. 2018;69(2):406-60

12. Bajaj JS, O'Leary JG, Reddy KR, Wong F, Biggins SW, Patton H, et al. Survival in infection-related acute-on-chronic liver failure is defined by extrahepatic organ failures. Hepatology. 2014;60(1):250-6.

13. Fernandez J, Acevedo J, Prado V, Mercado M, Castro M, Pavesi M, et al. Clinical course and short-term mortality of cirrhotic patients with infections other than spontaneous bacterial peritonitis [J]. Liver Int. 2017;37(3):385-95.

14. Cai J, Zhang M, Han T, Jiang HQ. Characteristics of infection and its impact on short-term outcome in patients with acute-on-chronic liver failure [J]. Medicine (Baltimore). 2017:96(37):e8057.

15. Wang C, Ma DQ, Luo S, Wang CM, Ding DP, Tian YY, et al. Incidence of infectious complications is associated with a high mortality in patients with hepatitis B virus-related acute-on-chronic liver failure [J]. World I Clin Cases. 2019:7(16):2204-16.

16. De Pauw B, Walsh TJ, Donnelly JP, Stevens DA, Edwards JE, Calandra T, et al. Revised definitions of invasive fungal disease from the European Organization for Research and Treatment of cancer/invasive fungal infections cooperative group and the National Institute of Allergy and Infectious Diseases mycoses study group (EORTC/MSG) consensus group [J]. Clin Infect Dis. 2008;46(12):1813-21.

17. Merli M, Lucidi C, Giannelli V, Giusto M, Riggio O, Falcone M, et al. Cirrhotic patients are at risk for health care-associated bacterial infections [J]. Clin Gastroenterol Hepatol. 2010;8(11):979-85.

18. Singer M, Deutschman CS, Seymour CW, Shankar-Hari M, Annane D, Bauer $\mathrm{M}$, et al. The third international consensus definitions for Sepsis and septic shock (Sepsis-3). Jama. 2016;315(8):801-10.

19. Kamath PS, Kim WR. The model for end-stage liver disease (MELD). Hepatology. 2007:45(3):797-805.

20. Kim WR, Biggins SW, Kremers WK, Wiesner RH, Kamath PS, Benson JT, et al. Hyponatremia and mortality among patients on the liver-transplant waiting list. N Engl J Med. 2008;359(10):1018-26.

21. Pugh RN, Murray-Lyon IM, Dawson JL, Pietroni MC, Williams R. Transection of the esophagus for bleeding oesophageal varices. Br J Surg. 1973;60(8): 646-9.

22. Jalan R, Saliba F, Pavesi M, Amoros A, Moreau R, Gines P, et al. Development and validation of a prognostic score to predict mortality in patients with acute-on-chronic liver failure. J Hepatol. 2014;61(5):1038-47. 
23. Vincent IL, de Mendonca A, Cantraine F, Moreno R, Takala J, Suter PM, et al. Use of the SOFA score to assess the incidence of organ dysfunction/failure in intensive care units: results of a multicenter, prospective study. Working group on "sepsis-related problems" of the European Society of Intensive Care Medicine. Crit Care Med. 1998;26(11):1793-800.

24. Zhao H, Shi Y, Dong H, Hu J, Zhang $X$, Yang M, et al. Community- or healthcare-associated bacterial infections increase long-term mortality in patients with acute Decompensation of cirrhosis. Am J Med Sci. 2018;355(2): $132-9$.

25. Martin-Mateos R, Alvarez-Mon M, Albillos A. Dysfunctional immune response in acute-on-chronic liver failure: it takes two to tango. Front Immunol. 2019; 10:973.

26. Piano S, Bartoletti M, Tonon M, Baldassarre M, Chies G, Romano A, et al. Assessment of Sepsis-3 criteria and quick SOFA in patients with cirrhosis and bacterial infections. Gut. 2018:67(10):1892-9.

27. Hung TH, Tseng CW, Hsieh YH, Tseng KC, Tsai CC, Tsai CC. High mortality of pneumonia in cirrhotic patients with ascites. BMC Gastroenterol. 2013;13:25.

28. Uvizl R, Kolar M, Herkel T, Vobrova M, Langova K. Possibilities for modifying risk factors for the development of hospital-acquired pneumonia in intensive care patients: results of a retrospective, observational study. Biomed Pap Med Fac Univ Palacky Olomouc Czech Repub. 2017:161(3):303-9.

29. Benson AB, Burton JR Jr, Austin GL, Biggins SW, Zimmerman MA, Kam I, et al. Differential effects of plasma and red blood cell transfusions on acute lung injury and infection risk following liver transplantation. Liver Transpl. 2011;17(2):149-58

30. Verma N, Singh S, Taneja S. Invasive fungal infections amongst patients with acute-on-chronic liver failure at high risk for fungal infections. Liver Int. 2019:39(3):503-13.

31. Lin $L N$, Zhu Y, Che FB, Gu JL, Chen JH. Invasive fungal infections secondary to acute-on-chronic liver failure: a retrospective study. Mycoses. 2013;56(4): 429-33.

32. Zang H, Liu F, Liu H, You S, Zhu B, Wan Z, et al. Incidence, risk factors and outcomes of acute kidney injury (AKI) in patients with acute-on-chronic liver failure (ACLF) of underlying cirrhosis. Hepatol Int. 2016;10(5):807-18.

33. Chen $\mathrm{N}$, Chen $\mathrm{X}$, Ding $\mathrm{X}$, Teng J. Analysis of the high incidence of acute kidney injury associated with acute-on-chronic liver failure. Hepatol Int. 2018;12(3):262-8.

\section{Publisher's Note}

Springer Nature remains neutral with regard to jurisdictional claims in published maps and institutional affiliations.

Ready to submit your research? Choose BMC and benefit from:

- fast, convenient online submission

- thorough peer review by experienced researchers in your field

- rapid publication on acceptance

- support for research data, including large and complex data types

- gold Open Access which fosters wider collaboration and increased citations

- maximum visibility for your research: over $100 \mathrm{M}$ website views per year

At $\mathrm{BMC}$, research is always in progress.

Learn more biomedcentral.com/submissions 